\title{
Recursos Tecnológicos e Ensino de Estatística na Educação Básica: um cenário de pesquisas brasileiras
}

Title: Technological Resources and Teaching Statistics in Basic Education: an overview of Brazilian research findings

Everton José Goldoni Estevam

Faculdade Estadual de Filosofia, Ciências e Letras de União da Vitória - FAFIUV/UNESPAR e PECEM/UEL evertonjgestevam@gmail.com
Marco Aurélio Kalinke

Universidade Tecnológica Federal do Paraná - UTFPR Campus de Curitiba e PPGECM/UFPR

kalinke@utfpr.edu.br

Resumo No presente artigo apresentamos um cenário de pesquisas brasileiras envolvendo recursos tecnológicos e o ensino de Estatística na Educação Básica, disponibilizadas no Banco de Teses da CAPES, na busca por compreender quais discussões têm sido privilegiadas. Enquanto estudo documental, realizamos uma análise interpretativa de 15 dissertações (2004-2011) e identificamos quatro eixos temáticos: (i) Softwares explorados em tarefas estatísticas; (ii) Conhecimentos estatísticos construídos elou mobilizados; (iii) Aspectos didáticos e metodológicos do uso da tecnologia no ensino de Estatística; e (iv) Perspectivas para Educação Estatística a Distância. Os resultados indicam que os recursos tecnológicos podem ampliar os limites do ensino de Estatística e possibilitam simulações e explorações que favorecem a compreensão de conceitos e habilidades que envolvem o processo de análise de dados, em detrimento de aspectos algébricos elou puramente estruturais.

Palavras-Chave: Educação Estatística, Tecnologias de Informação e Comunicação, Softwares, Probabilidade e Estatística.

\begin{abstract}
In this paper we show an overview of Brazilian research findings involving technological resources and teaching statistics in basic education, available in CAPES' thesis database, seeking to understand what discussions have been privileged. While documentary study, we did an interpretive analysis of 15 dissertations (2004-2011) and we identified four themes: (i) Software explored in tasks statistics, (ii) Statistical knowledge constructed and mobilized, (iii) educational and methodological aspects of use technology in teaching statistics, and (iv) Prospects for distance learning in Statistics Education. The results indicate that technological resources can expand the boundaries of teaching statistics and enable simulations and explorations that contribute to the understanding of concepts and skills involving the process of data analysis, rather than aspects of algebraic or purely structural.
\end{abstract}

Keywords: Statistics Education, Information and Communication Technology, Software, Probability and Statistics. 


\section{Introdução}

O debate quanto à utilização de tecnologias no processo de educação formal não é recente, tampouco simples. Particularmente, nos últimos tempos, é possível perceber alguns contornos mais bem definidos que privilegiam as Tecnologias de Informação e Comunicação (TIC) e, mais especificamente ainda, os recursos computacionais. Nos dois extremos dessa discussão estão aqueles que advogam que o computador pode substituir o professor e/ou incentivar os alunos à apatia em meio ao processo de construção de seu conhecimento, sob a crença de que tal tecnologia oferece a resposta pronta; e outros que assumem a associação da informática e dos recursos a ela relacionados como um avanço importante para o sistema educacional, já que possibilita a alunos e professores acesso às ferramentas tecnológicas atuais, ao mesmo tempo em que corrobora o exercício da função social da escola de preparar cidadãos e profissionais conscientes das exigências que os rodeiam e do contexto social no qual se inserem.

Em face desse debate, apropriamo-nos inicialmente dos pressupostos de Lévy [18] para sustentar nossa posição. Ele afirma que ao longo da história da humanidade, o conhecimento sofreu transformações decorrentes do surgimento de diferentes mídias como a fala, a escrita e, agora, a informática. Nesse contexto é que o pesquisador assegura que atualmente a informática amplia nossa memória e modifica o conhecimento produzido.

Kenski [17] corrobora essa ideia ao afirmar que desde o início da civilização é possível notar o predomínio de um determinado tipo de tecnologia que transforma as formas de organização social, a comunicação, a cultura e a própria aprendizagem de todo um grupo. Destarte, entendemos o computador e os recursos a ele associados como a "tecnologia" que caracteriza a sociedade atual e que fundamenta os diferentes comportamentos pessoais e sociais, amplamente discutidos e explorados nos diversos contextos e grupos. Assumimos, portanto, que a evolução e a presença dessas tecnologias originaram a necessidade de reorganização da atividade humana e o aparecimento de novas formas de mediação. Na sociedade hodierna, o computador, como uma ferramenta da atividade mental, transforma essa atividade, à medida que proporciona feedbacks a passos intermediários da ação humana, os quais dificilmente seriam dados por observadores externos [22]. Tal aspecto é relevante para diversos setores, sobretudo, para o contexto educacional.

Por outro lado, e relacionado a uma esfera específica da educação formal - a da Matemática - a Estatística consolida-se como campo expressivo do currículo formal por ser considerada ferramenta necessária à compreensão do contexto social em que o sujeito vive. Tal pressuposto sustenta as orientações curriculares brasileiras que sugerem considerá-la como essencial para a constituição de uma atitude crítica sobre aspectos sociais, políticos, culturais e científicos [8]. Esta ideia está sustentada nas características que distinguem essa ciência de outras. Por meio de suas ferramentas de representação e análise inferencial, ela possibilita a compreensão e o estudo de fenômenos que envolvem grande quantidade de dados, variabilidade e incertezas, fenômenos estes cada vez mais presentes no cotidiano das pessoas.

Assim, acreditamos que as tecnologias - aqui entendidas como as TIC - podem caracterizar uma ferramenta de apoio poderosa ao ensino de Estatística, uma vez que suas características podem favorecer a tomada de consciência e compreensão de determinados conceitos estatísticos e probabilísticos. Além disso, as TIC constituem recursos diligentes, que viabilizam e agilizam os processos de investigação e análise de dados.

Isso posto, e com o objetivo de compreender quais discussões têm sido privilegiadas nas pesquisas brasileiras envolvendo recursos tecnológicos e o ensino de Estatística na Educação Básica, realizamos um levantamento, junto ao Banco de Teses da Coordenadoria de Aperfeiçoamento de Pessoal de Nível Superior (CAPES) $)^{1}$, de dissertações e teses produzidas nessa temática. Os resultados desse levantamento ${ }^{2}$ apontaram 15 trabalhos, os quais subsidiam as análises que apresentamos no presente artigo. A seguir fazemos uma breve discussão sobre tecnologia e Educação Estatística, apresentamos os procedimentos metodológicos, exploramos alguns eixos de análise e, para finalizar, sintetizamos algumas considerações com nossas impressões quanto às pesquisas analisadas.

\section{A Tecnologia e a Construção de Conheci- mento Estatístico}

Diversos são os pesquisadores que apostam na utilização de tecnologias para o aprimoramento dos mecanismos de ensino e aprendizagem de Estatística, embora tal entendimento não seja consensual a este grupo, tampouco entre professores.

Freitas [14] afirma que calculadoras gráficas podem ser empregadas no ensino de Estatística para automatizar os cálculos e desencadear a formulação de conjecturas. Semelhantemente, Canavarro [7] salienta que esses equi-

\footnotetext{
${ }^{1}$ http://www.capes.gov.br/serviços/banco-de-teses.

${ }^{2}$ O levantamento foi realizado no segundo semestre do ano de 2012, considerando, portanto, os trabalhos realizados entre 2004 (primeiro trabalho encontrado) e 2011.
} 
pamentos possibilitam rapidez e rigor na produção de medidas Estatísticas. Isso possibilita maior empenho e atenção nos aspectos mais elaborados e complexos do trabalho, como na interpretação, organização e simplificação, o que privilegia a exploração dos dados e a investigação de hipóteses e conjecturas.

Nessa mesma perspectiva, Cobb [10] sustenta que os computadores libertam-nos para que possamos analisar dados reais com mais ênfase na interpretação do que nos cálculos, com vistas a priorizar as ideias de produção de dados aleatórios junto com a análise, com a inferência desses dados e com menor ênfase aos mecanismos das resoluções.

Segundo Batanero [2], o dinamismo, a velocidade e a crescente quantidade de novos softwares que permitem explorar todos os aspectos do processamento de dados justificam a pertinência das TIC no trabalho com Probabilidade e Estatística e possibilitam agregar novos tópicos ao ensino de Estatística. Nesse sentido, Batanero, Godino e Cañizares [3], salientam que os computadores oferecem aos estudantes uma variedade de ferramentas para simulação que auxiliam na exploração de conceitos e ideias que de outra forma seriam muito mais limitados ou até mesmo inviáveis. A título de exemplo, os autores citam a compreensão intuitiva de princípios probabilísticos. Borovcnik e Kapadia [5] também defendem a simulação enquanto estratégia de ensino e afirmam que, quando associada ao uso da tecnologia, ela auxilia na redução de cálculos técnicos, ao mesmo tempo em que possibilita ao aluno dedicar atenção aos conceitos em discussão.

Para Ponte, Brocardo e Oliveira [21] "as novas tecnologias não são apenas uma ferramenta útil para o trabalho em Estatística", mas "constituem mesmo um elemento indispensável na prática desse campo".

Segundo Burril [6], apesar de diversos desafios a serem enfrentados e questões delicadas a serem mais bem exploradas, é claro que a tecnologia faz a diferença na educação estatística, pois torna acessível a todos os alunos a Estatística e o raciocínio estatístico, fornece pistas sobre como trabalhar com dados e torna possível a utilização de dados reais e situações de interesse que motivam estudos estatísticos e matemáticos. Para o autor, a tecnologia amplia os limites da ciência estatística e, embora os desafios sejam grandes, uma questão é clara: “conteúdos do passado com ferramentas do passado não podem preparar os alunos de hoje para o mundo de amanhã" [6].

Chance, Ben-Zvi, Garfield e Medina [9] concordam com Burril, mas chamam atenção para o fato de que a utilização eficaz da tecnologia requer um planejamento cuidadoso, criatividade e entusiasmo. Apesar das capacidades infinitas que a tecnologia oferece, os professores devem ter cuidado com o uso de softwares sofisticados, pois eles podem resultar num esforço demasiado para aprender a usá-lo, quando o interesse maior está na sua aplicação nos contextos educacionais de interesse. A escolha de uma ferramenta tecnológica em particular deve ser feita com base na facilidade de uso, interatividade, ligações dinâmicas entre dados-gráficos-análises e um último aspecto relacionado à portabilidade. Boas escolhas podem favorecer a colaboração entre os alunos e as interações entre alunos e professor. Além disso, muitas vezes pode ser necessária uma combinação de várias ferramentas diferentes para se atingir determinados objetivos.

Esses apontamentos em âmbito internacional desafiam-nos a investigar em que medida as pesquisas brasileiras aproximam-se ou não dessas ideias, visando a verificar quais questões têm sido privilegiadas em nosso país. É isso que apresentamos a seguir.

\section{Procedimentos Metodológicos}

Para delinear o conjunto de trabalhos objeto de análise deste artigo, utilizamos os objetivos e/ou as questões de investigação presentes nos resumos das dissertações e teses disponíveis no Banco de Teses da CAPES, produzidas de 2004 (primeiro trabalho encontrado) a 2011. Foram selecionados todos os trabalhos relacionados a ensino que envolviam de maneira articulada "Tecnologia", "Probabilidade" e "Estatística", termos utilizados no campo "Assunto" na base de dados da CAPES. Cabe salientar que em diversos trabalhos as informações disponibilizadas nos resumos não ofereceram os elementos necessários ao nosso estudo, o que nos obrigou a buscar dados complementares no trabalho completo, geralmente encontrados na introdução e/ou no delineamento metodológico.

Como resultado desse mapeamento, identificamos 15 trabalhos listados no Quadro 1, em ordem cronológica de publicação. Não foi encontrada nenhuma tese de doutorado envolvendo a temática. Dessa forma, o código indicado na primeira coluna é composto pela letra $\mathrm{D}$, indicando tratar-se de dissertações de mestrado, e um número de ordem de 01 a 15. Este código foi utilizado para associar, posteriormente, cada trabalho com os eixos temáticos identificados. 


\begin{tabular}{|c|c|c|}
\hline Trabalho & Ano & Referência \\
\hline D01 & 2004 & $\begin{array}{l}\text { RICARDO, Vilson Wronscki. Metodologia de gerenciamento de base de dados para ensino-aprendizagem de estatística na } \\
\text { web. Dissertação (Mestrado em Ciência da Computação). Universidade Federal de Santa Catarina, 2004. } 108 \text { f. Orientador: } \\
\text { Silvia Modesto Nassar. }\end{array}$ \\
\hline D02 & 2005 & $\begin{array}{l}\text { PICHLER, Endineia. Metodologia de ensino/ aprendizagem de conceitos de probabilidade e estatística através de um sistema } \\
\text { tutor inteligente. Dissertação (Mestrado em Ciência da Computação). Universidade Federal de Santa Catarina, } 2005.97 \text { f. } \\
\text { Orientador: Marcelo Menezes Reis. }\end{array}$ \\
\hline D03 & 2006 & $\begin{array}{l}\text { TONI, Marijane Paese de. A compreensão da Estatística a partir da utilização da planilha. Dissertação (Mestrado em Educa- } \\
\text { ção em Ciências e Matemática). Pontifícia Universidade Católica do Rio Grande do Sul, } 2006.159 \text { f. Orientador: Lorí Viali. }\end{array}$ \\
\hline D04 & 2008 & $\begin{array}{l}\text { BRAGA, Julio César Pereira. O uso da planilha eletrônica como ferramenta na Matemática do ensino médio do Centro } \\
\text { Federal de educação Tecnológica de Januária - MG. Dissertação (Mestrado em Educação Agrícola). Universidade Federal } \\
\text { Rural do Rio de Janeiro, Seropédica, 2008. } 83 \text { f. Orientador: Marcelo Almeida Bairral. }\end{array}$ \\
\hline D05 & 2008 & $\begin{array}{l}\text { SILVA, Edgard Dias da. Os conceitos elementares de Estatística a partir do Homem Vitruviano: uma experiência de ensino } \\
\text { em ambiente computacional. Dissertação (Mestrado Profissional em Ensino de Matemática), Pontifícia Universidade Católica } \\
\text { de São Paulo, São Paulo, 2008. } 157 \text { f. Orientador: Sandra Maria Pinto Magina. }\end{array}$ \\
\hline D06 & 2008 & $\begin{array}{l}\text { VIEIRA, Márcia. Análise Exploratória de Dados: uma abordagem com alunos do ensino médio. Dissertação (Mestrado em } \\
\text { Ensino de Matemática). Pontifícia Universidade Católica de São Paulo, São Paulo, 2008. } 184 \text { f. Orientador: Cileda de Queiroz } \\
\text { e Silva Coutinho. }\end{array}$ \\
\hline D07 & 2009 & $\begin{array}{l}\text { LIMA, Osmar Antonio de. Distribuição Normal: uma introdução voltada ao ensino médio por simulações via planilha eletrô- } \\
\text { nica e exercícios interativos. Dissertação (Mestrado Profissional em Ensino de Matemática). Pontifícia Universidade Católica } \\
\text { de São Paulo, São Paulo, 2009. } 110 \text { f. Orientador: Cileda de Queiroz e Silva Coutinho. }\end{array}$ \\
\hline D08 & 2009 & $\begin{array}{l}\text { SOUZA, Leandro de Oliveira. A Educação Estatística no Ensino Fundamental e os Recursos Tecnológicos. Dissertação } \\
\text { (Mestrado em Ensino de Ciências e Matemática). Universidade Cruzeiro do Sul, São Paulo, 2009. } 192 \text { f. Orientador: Celi } \\
\text { Espasandin Lopes. }\end{array}$ \\
\hline D09 & 2010 & $\begin{array}{l}\text { DUARTE, Lucas Rodrigues. A utilização do software GeoGebra no ensino da distribuição Normal de Probabilidade: uma } \\
\text { aproximação entre a Geometria Dinâmica e a Educação Estatística. Dissertação (Mestrado em Ensino de Ciências e Matemá- } \\
\text { tica). Pontifícia Universidade Católica de Minas Gerais, Belo Horizonte, 2010. } 129 \text { f. Orientador: Dimas Felipe de Miranda. }\end{array}$ \\
\hline D10 & 2010 & $\begin{array}{l}\text { ESTEVAM, Everton José Goldoni. (Res)Significando a Educação Estatística no Ensino Fundamental: análise de uma se- } \\
\text { quência didática apoiada nas Tecnologias de Informação e Comunicação. Dissertação (Mestrado em Educação). Universidade } \\
\text { Estadual Paulista, Presidente Prudente, 2010. } 211 \text { f. Orientador: Monica Fürkotter. }\end{array}$ \\
\hline D11 & 2010 & $\begin{array}{l}\text { SAMPAIO, Luana Oliveira. Educação Estatística Crítica: uma possibilidade? Dissertação (Mestrado em Educação Matemáti- } \\
\text { ca). Universidade Estadual Paulista, Rio Claro, 2010. } 112 \text { f. Orientador: Maria Lucia Lorenzetti Wodewotzki. }\end{array}$ \\
\hline D12 & 2010 & $\begin{array}{l}\text { LIRA, Olga Cristina Teixeira. Uso de ferramentas do software Tinkerplots para interpretação de dados. Dissertação (Mestra- } \\
\text { do em Educação). Universidade Federal de Pernambuco, Recife, 2010. Orientador: Carlos Eduardo Ferreira Monteiro. }\end{array}$ \\
\hline D13 & 2011 & $\begin{array}{l}\text { FERREIRA, Robson dos Santos. Ensino de probabilidade com o uso do programa estatístico R numa perspectiva construcio- } \\
\text { nista. Dissertação (Mestrado em Educação Matemática). Universidade Bandeirante de São Paulo, São Paulo, } 2011.155 \text { f. } \\
\text { Orientador: Verônica Yumi Kataoka. }\end{array}$ \\
\hline D14 & 2011 & $\begin{array}{l}\text { ALVES, Iane Maria Pereira. A interpretação de gráficos em um ambiente computacional por alunos de uma escola rural do } \\
\text { municipio de Caruaru - PE. Dissertação (Mestrado em Educação Matemática e Tecnológica). Universidade Federal de Per- } \\
\text { nambuco, Recife, 2011. } 165 \text { f. Orientador: Carlos Eduardo Ferreira Monteiro. }\end{array}$ \\
\hline D15 & 2011 & $\begin{array}{l}\text { AMARANTE, Andreika Asseker. O uso do Tinkerplots para exploração de dados por professores de escolas rurais. Disserta- } \\
\text { ção (Mestrado em Educação Matemática e Tecnológica). Universidade Federal de Pernambuco, Recife, 2011. } 165 \text { f. Orienta- } \\
\text { dor: Carlos Eduardo Ferreira Monteiro. }\end{array}$ \\
\hline
\end{tabular}

Quadro 1 : Dissertações selecionadas do Banco de Teses da CAPES e analisadas no presente trabalho.

Para o estudo documental dos 15 trabalhos estabelecemos 5 descritores que auxiliaram na organização e delineamento da análise (Quadro 2). Todas as informações necessárias a cada descritor foram obtidas no próprio trabalho. Os descritores constituem dois eixos de interesse, um com informações a respeito da pesquisa e outro com relação aos seus participantes.

\begin{tabular}{|l|l|}
\hline \multicolumn{1}{|c|}{ Descritor } & \multicolumn{1}{c|}{ Definição } \\
\hline Autor e data & Sobrenome do autor da pesquisa e ano \\
\hline Objetivo(s) & Objetivo(s) da pesquisa \\
\hline Problema & Questão(ões) geral(ais) de investigação \\
\hline Resultados e conclusões & $\begin{array}{l}\text { Resultados alcançados em relação aos } \\
\text { objetivos }\end{array}$ \\
\hline Participantes & Sujeitos investigados \\
\hline
\end{tabular}

Quadro 2: Descritores utilizados para orientar a análise.
Com esse quadro foi possível constituir unidades de análise que deram origem aos eixos temáticos das pesquisas a respeito dos recursos tecnológicos e o ensino de Estatística. As convergências percebidas geraram quatro eixos, caracterizados a seguir e discutidos na próxima seção.

E1 - Softwares explorados em tarefas estatísticas: praticamente todos os trabalhos analisados (D02, D03, D04, D05, D06, D07, D08, D09, D10, D12,D13, D14, D15) envolveram a exploração de tarefas com conteúdos estatísticos realizadas com o auxílio de softwares, sejam educacionais ou não, específicos de Estatística ou não. Dessa forma, o primeiro eixo de análise tem por objetivo identificá-los e apresentar uma breve caracterização de 
cada um dos softwares explorados.

E2 - Conhecimentos estatísticos construídos elou mobilizados: são discutidos os conhecimentos estatísticos e probabilísticos explorados nas pesquisas analisadas. Integram o presente eixo as pesquisas D03, D04, D05, D06, D07, D09, D10, D12, D13, D14, D15.

E3 - Aspectos didáticos e metodológicos do uso da tecnologia no ensino de Estatística: considerando que o tipo de uso que se faz da tecnologia influencia significativamente a efetividade das tarefas propostas, o presente eixo explicita considerações relacionadas aos aspectos didáticos e metodológicos, a partir de situações discutidas nos trabalhos D03, D04, D05, D06, D07, D08, D09, D10, D11, D12, D13.

E4 - Perspectivas para Educação Estatística a Distância: alguns dos trabalhos analisados (D01, D02, D11) remetem a aspectos da Educação a Distância, que serão discutidos nesse $4^{\circ}$ eixo.

\section{Traçando um Cenário de Pesquisas Brasileiras}

Discutimos a seguir cada um dos eixos temáticos e os elementos presentes nos trabalhos a eles associados.

\subsection{Softwares explorados em tarefas estatísti- cas}

Considerando a temática do presente trabalho, a grande maioria das pesquisas analisadas envolveu a elaboração, realização e análise de tarefas que compreendiam a exploração de conceitos e ideias estatísticas em softwares, sendo alguns de caráter educacionais e outros não, alguns específicos de Estatística e outros não. O quadro 3 descreve os softwares utilizados e os respectivos trabalhos associados.

\begin{tabular}{|l|l|}
\hline \multicolumn{1}{|c|}{ Softwares } & \multicolumn{1}{c|}{ Trabalhos associados } \\
\hline Microsoft Excel & $\mathrm{D} 03, \mathrm{D} 04, \mathrm{D} 05, \mathrm{D} 07, \mathrm{D} 08, \mathrm{D} 10$ \\
\hline TinkerPlots & $\mathrm{D} 12, \mathrm{D} 14, \mathrm{D} 15$ \\
\hline Fathom & $\mathrm{D} 06, \mathrm{D} 08$ \\
\hline Tabletop & $\mathrm{D} 05$ \\
\hline GeoGebra & $\mathrm{D} 09$ \\
\hline$R$ & $\mathrm{D} 13$ \\
\hline SuperLogo & $\mathrm{D} 10$ \\
\hline
\end{tabular}

Quadro 3: Softwares explorados nos trabalhos analisados.

Dentre os softwares identificados no Quadro 3, o $\mathrm{Mi}$ crosoft Excel ganha destaque, uma vez que cinco trabalhos (D03, D04, D07, D08, D10) utilizaram-no em suas investigações. Trata-se de um aplicativo comercial Windows que integra o pacote Office e que, apesar de não ser um software direcionado a ambientes educacionais, parece ser bastante utilizado nas escolas por ser uma planilha eletrônica que fornece ferramentas para efetuar cálculos através de fórmulas e funções, construir planilhas e representações gráficas e analisar dados. Segundo D10, são cinco suas principais funções:

Planilhas: é possível armazenar, manipular, calcular e analisar dados tais como números, textos e fórmulas. Além disso, o Microsoft Excel possibilita a utilização de formatos pré-definidos em tabelas.

Bancos de dados: permite classificar, pesquisar e administrar facilmente uma grande quantidade de informações utilizando operações padronizadas de bancos de dados.

Gráficos: é possível rapidamente apresentar de forma visual uma representação de dados. Além de escolher tipos pré-definidos de gráficos, há a possibilidade de personalizar qualquer gráfico da maneira desejada.

Apresentações: o Microsoft Excel permite usar estilos de células, ferramentas de desenho, galeria de gráficos e formatos de tabela para criar apresentações de alta qualidade.

$\operatorname{Macros}^{3}$ : as tarefas que são frequentemente utilizadas podem ser automatizadas pela criação e armazenamento de suas próprias macros.

Quanto à utilização desse software no processo de ensino, D03 salienta que a "planilha é uma ferramenta que facilita a reflexão e a expressão do raciocínio, porém é preciso utilizar um material potencialmente significativo e do interesse dos estudantes, para elaborar as atividades". Enquanto vantagens, a pesquisadora destaca que o Microsoft Excel pode favorecer o trabalho cooperativo e colaborativo, evidenciado na ajuda mútua durante a resolução de problemas; desenvolver a autonomia na realização das tarefas, permitindo a exploração de forma abrangente de comandos e funções, o que proporciona novas descobertas; contribuir para a persistência dos estudantes diante de eventuais dificuldades; motivar os estudantes para a aprendizagem; e desenvolver a capacidade de aprender coisas novas de forma autônoma.

De modo geral, os trabalhos apontam que o uso da planilha eletrônica possibilita a automatização de cálculos e procedimentos e a priorização das discussões, análises e compreensão dos conceitos estatísticos envolvidos (D04, D07, D10). Contudo, D08 salienta que, por não favorecer o trabalho com variáveis, para construir um gráfico no

\footnotetext{
${ }^{3}$ Macro, na Ciência da Computação, é uma abstração que define como um padrão de entrada deve ser substituído por um padrão de saída, de acordo com um conjunto de regras.
} 
Microsoft Excel, o aluno deve saber muito bem o que deseja antes de começar, de modo que o resultado obtido corresponda àquele esperado inicialmente.

Três dos trabalhos analisados (D12, D14, D15) utilizaram o software TinkerPlots, um aplicativo educacional de análise de dados, desenvolvido na Universidade de Massachusetts, para o trabalho com estudantes dos anos iniciais do Ensino Fundamental (EF). Segundo seus autores, esse software é uma complexa ferramenta de ensino de Estatística que motiva o aluno a realizar atividades dentro da sala de aula e fora dela (D14). O TinkerPlots possui um ambiente dinâmico, no qual os estudantes podem organizar e explorar diferentes representações gráficas de dados. A tela inicial do software é constituída por uma área em branco, sem muitos atrativos visuais. A barra de menu é no idioma inglês e apresenta cinco ferramentas básicas: Cards, Table, Plot, Slider e Text. A função da ferramenta Cards é possibilitar o registro para criação de banco de dados da pesquisa a ser realizada. Ao ativar a ferramenta Table, automaticamente, obtém-se a distribuição dos dados em forma de tabela. A ferramenta Plot permite realizar a manipulação dos dados, que poderão ser analisados de acordo com suas ocorrências. O ícone Slider refere-se a um recurso pelo qual são realizadas alterações na amostra dos dados a serem trabalhados. Já a ferramenta Text, ao ser ativada, disponibiliza na tela uma caixa de texto na qual podem ser digitadas informações complementares ao trabalho que está sendo desenvolvido (D12).

Segundo D12 e D14, um elemento importante das explorações realizadas no TinkerPlots é a possibilidade de construção de variadas representações que não são normalmente propostas nos contextos escolares de tratamento de dados. Segundo D12, a partir das análises dos trabalhos desenvolvidos durante a pesquisa, foi possível identificar que essa flexibilização das maneiras de representar os dados também favoreceu diferentes possibilidades de pensar sobre eles, além de possibilitar um aproveitamento melhor do tempo.

Semelhantemente, D15 salienta que a manipulação de diferentes formas de representação dos dados proporcionou em muitas ocasiões uma mudança na reflexão e uma contribuição para a interpretação, oferecendo diferentes pistas na construção da resposta. Particularmente, tanto D14 quanto D15 destacam as ferramentas separar (separate) e gradiente de cores da função Plot, as quais, de acordo com a segunda pesquisadora, possibilitaram a percepção dos valores dispostos na escala, o que auxiliou no processo de interpretação dos dados.

O Fathom é outro software de estatística dinâmica com finalidades de ensino que também possibilita diferentes representações para um mesmo conjunto de dados, uma vez que, a conversão entre os diferentes registros ocorre de forma rápida e dinâmica, inibindo dificuldades operatórias e obstáculos didáticos. O software utiliza a língua inglesa e, de acordo com D06, suas características essenciais fundamentam-se na possibilidade de mover objetos, de representar e coordenar dados em tabelas e gráficos simultaneamente destacando suas características mais importantes, além do acesso imediato a uma série de funções que fornecem ampla flexibilidade para o trabalho de análise estatística.

D05 utilizou o software Tabletop, que foi desenvolvido com finalidade educacional para manipulação de dados e permite incluir as etapas de construção, exploração e análise de um banco de dados. De acordo com o pesquisador, o Tabletop apresenta cinco representações de dados: tabelas, caixas de pontos, diagrama de Venn, gráfico de pontos e histograma. Conta-se ainda com o modo de questionário. Estas facetas acopladas com as opções de títulos, ícones e marcação de grupos permitem aos estudantes tratar e visualizar os dados de diferentes formas. O potencial de animação do computador é aproveitado em várias representações visuais dos dados no Tabletop, já que quando o aluno seleciona as variáveis os ícones se movimentam e se agrupam de acordo com elas. Tais característica podem servir como uma ponte entre o 'concreto' e o 'abstrato', facilitando o estabelecimento de relações entre as informações advindas do mundo ao redor e os conceitos e tratamentos matemáticos dados a elas. Tendo por base a organização de um banco de dados no Tabletop, o aluno pode construir três tipos de gráficos: o Diagrama de Venn, o Gráfico de Frequência ou o Gráfico de Dupla Entrada.

Em uma outra perspectiva, D09 discute a utilização do GeoGebra para o ensino da distribuição normal de probabilidade. Trata-se de um software livre de matemática dinâmica que associa geometria, álgebra e cálculo. O GeoGebra fornece três diferentes campos para os objetos matemáticos: a Zona Gráfica, a Zona Algébrica (ou numérica) e a Folha de Cálculo. Elas permitem mostrar os objetos matemáticos em três diferentes representações: graficamente (por exemplo, pontos, gráficos de funções), algebricamente (por exemplo, coordenadas de pontos, equações) e nas células da folha de cálculo. Assim, todas as representações do mesmo objeto estão ligadas dinamicamente e se adaptam automaticamente às mudanças realizadas em qualquer uma delas, independentemente da forma como esses objetos foram inicialmente criados. Segundo D09, os dados analisados evidenciaram o quanto é importante a representação geométrica para compreensão da distribuição normal de probabilidade, bem como as contribuições confluentes do software que "ao mesmo tempo permite observar melhor as representações equivocadas, ajudando a identificar o conflito cognitivo, e assim, possibilita ao professor controlar e entender melhor a atividade mental do aluno". 
Já D13 utiliza o software $R$ para o ensino de Probabilidade. $\mathrm{O} R$ é uma linguagem orientada a objetos criada em 1996 que, aliada a um ambiente integrado, permite a manipulação de dados, realização de cálculos e geração de gráficos. Por ser um software livre, o software $R$ é considerado uma importante ferramenta na análise e na manipulação de dados, com testes paramétricos e não paramétricos, modelagem linear e não linear, análise de séries temporais, análise de sobrevivência, simulação e estatística espacial, entre outros. Apresenta ainda facilidade na elaboração de diversos tipos de gráficos, cujo usuário tem pleno controle sobre o gráfico criado.

Por suas características, os comandos em $R$ são dados na forma de linguagem de programação, na janela console que se abre ao iniciar o software. Contudo, a partir da versão 2.1.1, ele passou a contar com o editor de script, que facilita a execução de comandos diretamente no software, quando comparado com a janela console. Segundo D13, o uso do computador por meio do software $R$ constituiu-se uma importante ferramenta, pois potencializou a visualização de experimentos probabilísticos por meio de simulações, o que proporcionou de forma significativa a observação, por exemplo, "do fenômeno de convergência, reflexão esta que já vinha sendo construída no decorrer do desenvolvimento do experimento e que ao final pôde ser concluída com um experimento com maior número de lançamentos".

O trabalho D10 utilizou o SuperLogo 3.0 para a construção de gráficos estatísticos. Trata-se de um software gráfico de domínio público, baseado na linguagem de programação Logo. Sua integração aos processos de ensino e aprendizagem visa a facilitar a intervenção do professor e a compreensão do raciocínio do aluno [24]. O registro dos comandos utilizados pelo aprendiz é o que diferencia o SuperLogo 3.0 de outros softwares, bem como do lápis e papel. Diferentemente da linguagem complexa do ambiente $R$, a linguagem de programação Logo é direcionada à movimentação de uma tartaruga (cursor gráfico) na janela gráfica, que pode ser feita por meio de comandos de deslocamento e giro semelhantes à língua natural: parafrente (ou pf), paratras (ou pt), paradireita (ou pd) e paraesquerda (ou pe). Além desses comandos, é necessário indicar o número de passos de tartaruga (unidade de medida do SuperLogo 3.0), no caso de deslocamento, ou o grau do giro. Segundo D10, apesar de algumas dificuldades iniciais, o uso do software possibilitou a compreensão das estruturas que constituem os gráficos, pois a construção de gráficos estatísticos em Logo exige a explicitação de conceitos subjacentes aos gráficos, diferentemente de outros softwares que realizam essa tarefa de maneira "automática".

Além desses softwares, D08 utiliza Tarefas e Objetos de Aprendizagem encontrados na web para discutir conceitos, estruturas e ideias estatísticas e probabilísticas.
Godino, Ruiz, Roa, Pareja e Recio [16] fazem uma análise sobre os recursos interativos para o ensino de Estatística disponíveis na internet. Segundo os pesquisadores, o número e a qualidade crescente desses recursos têm facilitado muito o ensino e a aprendizagem estatística. Dentre eles, importantes recursos, tanto para o Ensino Fundamental (EF) quanto para o Ensino Médio (EM), têm sido desenvolvidos pelo National Council of Teachers of Mathematics (NCTM) e podem facilmente ser encontrados em seu site ${ }^{4}$, onde há ferramentas para diferentes abordagens dos conteúdos matemáticos, estatísticos de análise e probabilísticos. Assim, D08 explora o ambiente Illuminations $^{5}$, que possibilita a interação com gráficos e foi projetado pelo NCTM para prover recursos didáticos, com o objetivo de contribuir para o ensino e aprendizagem. $\mathrm{O}$ Spinners $^{6}$, que constitui um simulador virtual em língua espanhola, inglesa e francesa e permite ensinar e discutir probabilidade e escolhas aleatórias. E um terceiro recurso, o Circle Graph ${ }^{7}$, que permite a exploração de gráficos de setores (ou pizza), em que os alunos podem utilizar conjuntos de dados predefinidos ou inserir seus próprios dados.

Um último software também discutido na pesquisa D08 é o Navigating through Probability Applets, que possibilita simulações probabilísticas. O programa pode ser encontrado no CD-ROM que acompanha o livro $\mathrm{Na}$ vigating Through Probability in Grades 6-8 [5].

Identificados os diversos softwares explorados nas pesquisas analisadas, julgamos pertinente investigar quais os conceitos estatísticos efetivamente mobilizados ou construídos com as tarefas desenvolvidas utilizando tais recursos, ideia que sustenta o próximo eixo de análise.

\subsection{Conhecimentos estatísticos construídos e/ou mobilizados}

D03, investigando se o uso de planilhas eletrônicas pode auxiliar na aprendizagem dos conteúdos de Estatística e despertar o interesse de alunos do terceiro ano de Ensino Médio (EM), abordou os conceitos de tabela de distribuição de frequências (amplitude, limites superior e inferior, frequência relativa e frequência acumulada) e discutiu o cálculo e interpretação de medidas de tendência central (média, moda e mediana) e dispersão (desvio médio, variância e desvio padrão). A autora salienta que o trabalho envolvendo o software Microsoft Excel mostrou-se adequado aos interesses didáticos da Estatística e afirma que:

\footnotetext{
${ }^{4}$ http://www.nctm.org

${ }_{6}^{5}$ http://illuminations.nctm.org

http://nlvm.usu.edu/en/nav/frames asid 186 g 1 t 5.html?open= activities

${ }^{7}$ http://www.shodor.org/interactivate/activities/CircleGraph/
} 
O uso da planilha poderá não provocar um aumento significativo em termos de aprendizagem, porém o fato de tornar as aulas agradáveis, desafiadoras e menos cansativas, como os próprios estudantes relataram, é um dos fatores primordiais para alcançarmos uma aprendizagem mais duradoura. (D03).

Semelhante constatação é encontrada em D04, quando o pesquisador buscou desenvolver atividades que promovessem habilidades na construção de conhecimentos matemáticos de alunos de um Curso Técnico em Agropecuário e Ensino Médio (EM). Trabalhando termos relacionados à pesquisa estatística (população, amostra, variáveis e tabela de frequências), gráficos de setores, segmentos, barras e histogramas, medidas de tendência central (média, moda e mediana) e dispersão (variância e desvio padrão), ele afirma que o Microsoft Excel potencializou a compreensão desses conceitos e, além disso, contribuiu "para o desenvolvimento da capacidade de raciocínio e resolução de problemas, de comunicação, bem como o espírito crítico e criativo e o estabelecimento de conexões entre diferentes temas matemáticos" [D04], competências desejáveis para este nível de ensino.

D05 investigou as potencialidades de uma intervenção de ensino sobre os conceitos elementares de Estatística com alunos do EM, construída a partir de uma visita cultural à exposição "O Homem Vitruviano", de Leonardo Da Vinci, e teve como ferramenta o ambiente computacional Tabletop. A investigação Estatística abordou a tabela de distribuição de frequências, a construção, análise e interpretação de medidas de tendência central (média, moda e mediana) e representações gráficas, sendo estas últimas realizadas no software estatístico. A possibilidade de identificar no gráfico o indivíduo que está sendo representado mostrou-se um facilitador das análises, assim como a automatização do cálculo das medidas de tendência central.

D06 optou por avaliar em que medida as articulações entre os diferentes tipos de registros de representações semióticas podem constituir acesso à compreensão em Estatística. Para tanto, utilizou o software Fathom no contexto de um processo investigativo envolvendo classificação de variáveis, representações gráficas (setores, colunas, barras, dot-plot ou gráfico de ponto, box-plot ou gráfico de caixas e histograma) e medidas resumo (média, moda, mediana e quartis).

O desenvolvimento da sequência didática mostrou que as interações dos diferentes tipos de representação contribuíram com a compreensão de conceitos como média aritmética e mediana e, também, para a análise e interpretação de gráficos de colunas e de pontos. No entanto, estas variáveis ainda foram insuficientes na compreensão de medidas como os quartis e do gráfico de caixas. A pesquisadora salienta a importância da articula- ção dos diferentes tipos de registro de representação semiótica, como entre tabelas e gráficos, representação numérica e gráfica de uma medida (como a média ou a mediana), para compreensão destes conceitos pelos alunos.

[...] A influência do ambiente computacional foi fundamental no sentido de possibilitar a visualização dos diferentes tipos de registro de representação semiótica de uma distribuição de frequências, principalmente no caso da representação gráfica de medidas como a média e a mediana, contribuindo para compreensão destes conceitos, como os quartis e a construção do Box-Plot, acreditamos que o uso desse ambiente possa contribuir com a compreensão de tais conceitos, desde que seja desenvolvida uma sequencia de atividades específica, com maior tempo de duração. [...] No entanto, nem sempre os alunos faziam uso da multiplicidade de informações/registros para a análise das atividades. (D06).

D13 investigou a aprendizagem de conceitos probabilísticos de alunos do $3^{\circ}$ ano do EM, por meio da aplicação do experimento de ensino "Passeios Aleatórios da Carlinha". Trata-se de um estudo que explorou os ambientes Papel e Lápis e o software $R$, sob a perspectiva do letramento probabilístico de Gal [15] e do construcionismo de Papert [20]. Especificamente, abordou os princípios teórico e frequentista de probabilidade, envolvendo tabelas de distribuição de frequências e gráficos de barras. Nas análises, o pesquisador afirma que o trabalho com um número maior de simulações que o recurso computacional possibilitou proporcionou reflexões diferentes daquelas usualmente desenvolvidas no ambiente Papel e Lápis, bem como a discussão do conceito de não equiprobabilidade. Embora o experimento tenha evidenciado dificuldades pontuais, a possibilidade de confronto entre a probabilidade frequentista e a teórica, proporcionou aos alunos novas reflexões em torno dos conceitos probabilísticos. Isso foi potencializada pelo experimento, bem como pelo uso do software $R$ (D13).

D07 buscou introduzir os primeiros conceitos relativos à distribuição normal no EM, ainda de forma intuitiva, utilizando como recurso didático uma planilha eletrônica (Microsoft Excel) e percebeu que os alunos passaram a reconhecer suas características e a forma de representação gráfica. Em síntese, o pesquisador afirma que o uso da planilha eletrônica possibilitou aos alunos identificar os conceitos que envolvem a distribuição normal, facilitando sua interação com os objetivos de estudo. Os alunos perceberam ainda a relação existente entre Estatística e Probabilidade, o que o pesquisador denominou como estocástica (D08).

Também com o objetivo de abordar a distribuição normal de probabilidade, porém num curso tecnológico 
associado ao EM e utilizando o software GeoGebra, D09 afirma que ele contribuiu com processo de experimentação e permitiu simulações e visualizações dos modelos. Essas características possibilitam trabalhos investigativos, exploratórios e problematizados na busca por regularidades e favorece tanto o processo de aprendizagem dos alunos quanto as intervenções do professor.

Já D10 se propôs a avaliar as características que podem contribuir para que uma investigação exploratória de dados represente uma situação-problema "facilitadora" no processo de ensino e aprendizagem, bem como, em que medida as TIC podem contribuir com esse processo. $\mathrm{O}$ pesquisador afirma que o Microsoft Excel favoreceu a organização de tabelas, em virtude de sua estrutura de planilha eletrônica dinâmica. No que concerne ao SuperLogo 3.0 para a construção de representações gráficas, apesar de a linguagem de programação utilizada apresentar-se inicialmente como um dificultador, após a familiarização dos alunos, a construção de gráficos por meio dos comandos em Logo revelou-se um impulsionador da atribuição de significado aos gráficos de colunas, barras, setores e histograma, na medida em que, para ensinar a tartaruga, foi necessário que o aluno raciocinasse sobre as relações e conceitos subjacentes às estruturas gráficas, de modo a indicar o comando correto (D10).

Os trabalhos D12, D14 e D15 investigaram as potencialidades e contribuições do software Tinkerplots para interpretação de gráficos por alunos do $7^{\circ}$ ano do EF, $5^{\circ}$ ano do EF e professores das séries iniciais, respectivamente. As análises evidenciaram que a possibilidade de diferentes representações para um conjunto de dados favoreceu o processo de exploração dos dados, a compreensão e a atribuição de significado a essas representações.

Identificados os conceitos abordados nas pesquisas analisadas, discutiremos a seguir os aspectos metodológicos que permeiam a utilização dos diferentes softwares, com vistas à promoção da aprendizagem desses conceitos.

\subsection{Aspectos didáticos e metodológicos do uso da tecnologia no ensino de Estatística}

Oliveira, Costa e Moreira [19] salientam que é pressuposto de qualquer discussão quanto ao uso de tecnologias na educação a ideia de que toda prática pedagógica reflete uma certa concepção sobre o ensinar e o aprender. Nesse sentido, o presente eixo explicita os aspectos didáticos e metodológicos presentes nos trabalhos analisados, com vistas a identificar os pressupostos assumidos no que se refere a ensinar e aprender Estatística a partir da exploração de recursos tecnológicos.

D10 e D13 assumem a perspectiva construcionista de
Papert [20], que tem origem no construtivismo piagetiano e concebe tarefas realizadas no computador como (res)significantes no processo de construção do conhecimento, ao mesmo tempo em que sugere uma redução no papel da instrução em meio ao processo de aprendizagem. "A atitude construcionista no ensino não é, em absoluto, dispensável por ser minimalista - a meta é ensinar de forma a produzir a maior aprendizagem a partir do mínimo de ensino" [20].

Na constituição de um ambiente educacional construcionista efetivo, é exigido muito mais do que um aprendiz e um computador. É essencial a estruturação de um ambiente que favoreça a motivação do aluno na busca pelo conhecimento, que incentive a discussão, a investigação e a descoberta. Nesse sentido, o computador pode favorecer uma sequência de ações que procura descrever a interação entre aluno-computador na resolução de um problema. Tal processo se dá por meio da espiral da aprendizagem descrição-execução-reflexão-depuração [23], que respeita as características específicas de cada um. Nesse contexto, o professor assume a difícil tarefa de mediar os processos, intervindo nos momentos em que percebe equívocos e/ou insuficiência dos conhecimentos, sem que essa intervenção comprometa a autonomia do aluno enquanto construtor de seu conhecimento. D10 explicita isso na afirmação de que "o aluno, diante de inconsistência entre a ação da tartaruga e a esperada ao indicar o comando, autonomamente refletia e depurava o erro, às vezes com a interação com outros colegas do grupo e dos demais grupos". Semelhantemente, D13 destaca que

[...] Foi possível notar que o trabalho propiciou importantes discussões referentes à comparação entre os resultados simulados e teóricos, a observação do fenômeno da convergência, dentre outras, como proposto nos objetivos descritos inicialmente. Além disso, o desenvolvimento desse trabalho fazendo uso da metodologia do Design Experiment ${ }^{8}$ permitiu ao aluno uma participação mais ativa no processo, e, por conseguinte, um ambiente de aprendizagem na perspectiva construcionista. (D13).

Sem assumir tais pressupostos, D09 assume as tarefas investigativas e a resolução de problemas como fatores que favorecem aspectos semelhantes à perspectiva construcionista, com vistas à compreensão e significação dos conceitos estatísticos em ambientes computacionais, no caso específico, a distribuição normal no GeoGebra.

A Modelagem Matemática na perspectiva da Educa-

\footnotetext{
${ }^{8}$ Trata-se de um modelo que assume que o desenvolvimento da pesquisa deve ocorrer por meio de ciclos contínuos de design, de interação, de análise e de redesign, que foca os sucessos e as falhas nas interações que contribuem para compreensão dos fatores de aprendizagem envolvidos a partir de relatos fidedignos sobre métodos que possam documentar e conectar processos de interações aos resultados de interesse (D13).
} 
ção Estatística Crítica é assumida por D11, salientando que a modelagem contribui para o estabelecimento de consistência às ideias e procedimentos estatísticos, ao mesmo tempo em que a Estatística favorece o processo de modelagem.

Como princípios de aprendizagem estatística com tecnologia destaca-se também a teoria dos Registros de Representação Semiótica de Duval [12, 13], a qual destaca que para que ocorra aprendizagem é necessário que o indivíduo utilize diferentes registros para representar um mesmo objeto, cuja conceituação só é alcançada com a articulação de diferentes registros de representação. D05 salienta que a utilização do Tabletop favoreceu a mobilização de diferentes registros e a superação da ideia de que a Estatística seja enfadonha e limitada a apenas cálculos algébricos. D06 ratifica essa ideia utilizando o Fathom e acrescenta a importância de essa mobilização estar articulada em uma sequência estruturada de atividades.

Pudemos perceber a importância da articulação dos diferentes tipos de registro de representação semiótica, como entre tabelas e gráficos, representação numérica e gráfica de uma medida como a média ou a mediana, para compreensão destes conceitos pelos alunos. (D06).

Complementarmente, D10 destaca os sentidos das conversões entre tabelas e gráficos, por exemplo, cuja predominância se dá na elaboração de gráficos a partir de tabelas, e isso não garante a compreensão e/ou relação quanto à elaboração de uma tabela a partir de informações expressas em gráficos.

Outro aspecto presente nos trabalhos analisados (D05, D06, D10) são os níveis de compreensão de informações expressas em gráficos de Curcio [11], a saber:

Ler os dados: este nível de compreensão requer uma leitura literal do gráfico. A interpretação da informação contida na representação não é realizada. O leitor retira os fatos explícitos, lendo apenas informações apresentadas nos eixos e nas legendas. Não realiza qualquer tipo de interpretação e responde apenas às questões imediatas.

Ler entre os dados: inclui a interpretação e integração dos dados do gráfico, requer habilidade para comparar quantidades e o uso de conceitos e destrezas matemáticas. O leitor realiza alguma interpretação dos dados e da forma como estes estão integrados no gráfico, recorrendo a outros conceitos e capacidades, que demandam domínio das relações matemáticas implícitas em uma estrutura gráfica. Nesse nível, têm início as inferências de natureza simples.

Ler além dos dados: requer que o leitor realize previsões e inferências a partir dos dados sobre informações que não estão presentes diretamente no gráfico. A pessoa é capaz de inferir ou predizer um determinado resultado ou acontecimento em função de vários conhecimentos e não apenas a partir das informações apresentadas. Ao atingir esse patamar, o leitor tem condição de responder a perguntas implícitas tendo como base extrapolações, previsões ou inferências realizadas a partir de uma interpretação.

Explicitamente, D10 salienta que os alunos foram capazes de ler os dados e, além disso, conseguiram realizar comparações e identificar as relações proporcionais existentes entre gráficos. Dessa forma, as atividades de construção das representações no SuperLogo 3.0 proporcionaram o desenvolvimento das habilidades de leitura entre os dados. Os alunos utilizaram conceitos matemáticos e estatísticos de maneira adequada, o que possibilitou a retirada de informações concisas das análises realizadas. Ficou evidente a evolução quanto à compreensão das diferentes formas de representação, sua aplicabilidade (ou não) e funcionalidade.

D05 apoia-se na Teoria dos Campos Conceituais de Vergnaud [25] para afirmar que

\begin{abstract}
Para que o aluno possa se apropriar dos invariantes operatórios dos diversos conceitos e procedimentos aqui trabalhados [tabela de distribuição de frequências, medidas de tendência central e gráficos estatísticos], é preciso que os professores venham construindo esses conceitos ao longo da instrução, através de suas propriedades, situações diversificadas (papel e lápis, ambiente computacional), envolvendo variáveis de diversas naturezas. (D05).
\end{abstract}

D03 assume a perspectiva de Aprendizagem Significativa de Ausubel [1] e salienta que o computador pode ser utilizado ou como meio de transmissão de conhecimentos ou para criação de ambientes de ensinoaprendizagem que enfatizam a construção do conhecimento através da iniciativa do educando, o que implicitamente remete aos pressupostos do construcionismo. Assim, destaca que não é produtivo o professor utilizar apenas as funções disponíveis do software quando se explora, por exemplo, planilhas eletrônicas no ensino da Estatística. É preciso pensar tarefas que favoreçam a apropriação significativa do conteúdo pelo aluno. "É preciso olhar o computador como um recurso que vai motivar o estudante contribuindo para uma aprendizagem significativa, pois o processo de descrever, refletir e pensar não acontece naturalmente quando o aluno é colocado na frente do computador" (D03).

D08 destaca que as atividades de ensino buscaram fazer com que a aprendizagem ocorresse por meio da internalização, a partir de um processo anterior, de troca, com dimensão coletiva e com apoio de recursos tecnológicos. "Tornou-se evidente que a inserção de tais recursos gera conhecimentos mais amplos e precisos, porém exige do 
professor um conhecimento teórico-metodológico muito mais aprofundado sobre o assunto" (D08).

Diversos são os trabalhos (D04, D05, D09, D10, D11, D12, D13) que apontam para a importância de se abordar conceitos estatísticos e probabilísticos, utilizando recursos tecnológicos ou não, a partir de um processo investigativo que se aproxime do modelo científico. Eles destacam que, mesmo que as situações exploradas se aproximem daquelas emergentes em contextos escolares, ao coletar e construir um banco de dados e as representações das informações, os estudantes estão atuando em contextos investigativos, nos quais produtores de dados interpretam representações construídas por eles mesmos (D13).

Ficou evidenciado, nesta pesquisa, que, através das quatro atividades desenvolvidas, conseguimos levar os alunos à construção e à simulação dos dados, de forma que conseguimos também desenvolver uma estatística, em que prevalece a análise crítica em detrimento de uma construção mecânica, em que não há espaço para o pensamento estatístico. (D08).

Embora essas orientações apliquem-se tanto a ambientes computacionais quanto a outros ambientes de ensino, os trabalhos destacam que a principal diferença entre essas duas abordagens é a possibilidade de mobilização de diferentes registros, sua manipulação dinâmica (D06, D08), bem como a automatização de cálculos e construções gráficas (D04, D07, D10).

\subsection{Perspectivas para Educação Estatística a Distância}

Dentre os trabalhos analisados, percebemos alguns que remetem a aspectos relacionados ao ensino de Estatística a distância (D01, D02, D11), não necessariamente voltados à Educação Básica, mas que julgamos pertinentes de serem abordados na presente discussão.

D01 desenvolve uma metodologia de gerenciamento de base de dados que permite ao usuário alterar suas próprias bases de dados em tempo real usando a Internet como meio de comunicação, a SEstatNet ${ }^{9}$. O pesquisador destaca que a metodologia procurou desenvolver uma interface gráfica semelhante a uma planilha que oferece recursos adicionais de gerência de dados pela web. Na idealização das interfaces foram considerados todos os conceitos estudados e a tendência atual no que diz respeito a sites educacionais.

Dentre os recursos adicionais implementados na planilha de dados, destacam-se a tipificação automática de variáveis (SETip) e facilidades de criação e

\footnotetext{
${ }^{9}$ http://www.sestatnet.ufsc.br/
}

transformação de colunas de dados com funções logarítmica, exponencial, inversa, raiz quadrada e a padronização pela normal, que são operações essenciais no ensino-aprendizagem de estatística. (D01).

Essa tipificação permite reconhecer o tipo de variável e classificá-la como qualitativa ou quantitativa, de acordo com alguns padrões descobertos nos dados, um aspecto extremamente importante para a tomada de decisões no processo de análise final. Trata-se de um ambiente de ensino-aprendizagem de Estatística que, segundo o pesquisador, foi validado por especialistas em análise de dados, mas que necessita ser legitimado por pesquisadores e usuários da área educacional.

Complementarmente, D02 elaborou uma metodologia que incluiu um sistema tutor para auxílio no ensino e na aprendizagem de conceitos de Probabilidade e Estatística (princípios de probabilidade, variáveis aleatórias discretas e variáveis aleatórias contínuas). Como resultado de suas análises, a pesquisadora afirma que a necessidade de ensinar/aprender de maneira alternativa e flexível é um dos fatores que incentivam o desenvolvimento de softwares para a educação. Ademais, a utilização de sistemas tutores inteligentes pode facilitar a inclusão dos aprendizes no mundo digital e auxiliar na "transmissão" de conhecimento.

Em virtude da dificuldade de se estruturar uma lógica de programação que possibilitasse a elaboração de exercícios pelos próprios alunos e posteriormente sua interpretação e resolução, o sistema é pautado em exercícios fixos envolvendo os conteúdos listados acima.

O sistema proposto apresenta o conteúdo e ao final traz exercícios do conteúdo para que o aprendiz perceba como está seu aprendizado. $\mathrm{O}$ aprendiz pode verificar se a resposta por ele encontrada está correta, solicitando ao sistema a verificação do exercício resolvido. Para o professor o sistema apresenta um relatório de acompanhamento, onde estão as lições acessadas por determinado aprendiz, permitindo avaliar como ele está se saindo, em quais lições há mais dificuldades, entre outras informações. (D02).

Por fim, ganha destaque a pesquisa D11, que não utiliza software específico de estatística, mas, buscando criar condições para que alunos de um curso de licenciatura tivessem uma formação Estatística mais crítica, investigou a natureza das discussões originadas num ambiente de Modelagem Matemática e analisou o papel de cada uma de suas classes. Para tanto, a pesquisadora lançou mão do ambiente Tidia $A e^{10}$, que segundo ela, configurou-se como um ambiente virtual de aprendizagem, mas cujas interações foram mais efetivas por meio

\footnotetext{
${ }^{10} \mathrm{http}: / /$ tidia-ae.usp.br
} 
do $\mathrm{MSN}^{11}$ e e-mail, observando as especificidades destes ambientes. $\mathrm{O}$ aspecto que fez ampliar as possibilidades nesta pesquisa, foi a interação online.

[...] concluímos que sem estes ambientes o projeto de Modelagem Matemática no Ensino de Estatística na busca pelo desenvolvimento de uma EEC [Educação Estatística Crítica], especificadamente nesta pesquisa, provavelmente não teria tido resultados significativos, pois a interação com os alunos apenas foi possibilitada diante da dinâmica a Distância, visto que a maior parte deles trabalhava no turno oposto e, por isso, tinha restrições de horários para encontros presenciais. (D11).

A pesquisadora conclui, portanto, que o desenvolvimento de uma Educação Estatística Crítica, por meio de práticas de Modelagem Matemática a Distância, não só é possível, como esse modelo apresenta vantagens significativas quando comparado ao ensino presencial.

\section{Considerações Finais}

Os trabalhos analisados discutem aspectos bastante diversos de Probabilidade e Estatística, bem como exploram diferentes softwares, com características e potencialidades particulares. Neste sentido, o presente trabalho parece contribuir para uma sistematização da produção brasileira (dissertações) relacionada aos recursos tecnológicos para o ensino de Estatística na Educação Básica, o que colabora para o delineamento de trabalhos futuros, bem como pode orientar professores que intentem explorar tarefas e softwares semelhantes em suas salas de aula. Cabe um destaque para o fato de não termos encontrado teses nesse campo de pesquisa. Considerando uma temática em franca evidência e expansão, acreditamos que pesquisas de doutorado podem (e devem) fazer discussões mais profundas e abrangentes, na busca por evidenciar possibilidades e limites dos recursos tecnológicos no ensino e na aprendizagem de Estatística, tanto na Educação Básica quanto na formação de professores.

No que concerne aos softwares identificados nos trabalhos analisados, é possível perceber que apesar de explorarem aplicativos específicos para o ensino de Estatística (Fathom, Tabletop e TinkerPlots), as pesquisas mostram possibilidades de adaptações de tarefas em softwares com outras características, mas que podem contribuir para a exploração de conceitos, ideias e habilidades estatísticas. O Microsoft Excel para exploração de planilhas eletrônicas dinâmicas; o ambiente $R$ para associação

\footnotetext{
${ }^{11}$ Abreviação de Microsoft Service Network que é um portal e uma rede de serviços oferecidos pela Microsoft em suas estratégias envolvendo tecnologias de Internet que possibilita a comunicação a partir de mensagens síncronas e assíncronas por meio da Internet.
}

de aspectos estatísticos e probabilísticos; o GeoGebra para o estudo da distribuição normal; e o SuperLogo para exploração de gráficos estatísticos, exemplificam muito bem a afirmação acima. Julgamos pertinente, portanto, destacar que mais importante que as características que o software apresenta para abordagem de conceitos estatísticos e probabilísticos é o modo como se planeja, executa e avalia tarefas explorando diferentes ambientes, a partir de objetivos específicos claros traçados inicialmente. Algumas dessas características foram apontadas no terceiro eixo de análise, cujos destaques revelam a necessidade de formação dos professores para explorarem de modo adequado essas ferramentas.

Por outro lado, um aspecto que chama a atenção é que a maioria dos softwares utilizados são comerciais, como é o caso do Microsoft Excel, TinkerPlots, Fathom e Tabletop. Considerando o ônus de tal característica aos sistemas públicos de ensino, o que pode até mesmo inviabilizar a realização em sala de aula de tarefas semelhantes às exploradas nas pesquisas, é necessário o desenvolvimento de softwares gratuito e/ou livres que possibilitem a exploração de tarefas estatísticas em quaisquer estabelecimentos, sem custos adicionais. Complementarmente, outro campo fértil de investigação refere-se às possibilidades de desenvolvimento de tarefas com softwares e ambientes já existentes e que não tenham fins comerciais. A utilização do Calc em substituição ao Microsoft Excel exemplifica uma possibilidade que corrobora nossa preocupação.

Quanto aos conceitos explorados, parece evidente que os recursos tecnológicos adentram as aulas de Estatística como alternativa para priorizar o raciocínio, a compreensão dos processos de análise de dados e dos conceitos que os permeiam, ao mesmo tempo em que apontam uma desvalorização dos cálculos algébricos e da repetição de procedimentos, sem finalidade relevante àquilo que se assume por Educação Estatística. Talvez este seja o grande diferencial dos recursos tecnológicos, quando comparados com outras alternativas didáticas e que justifica sua pertinência ao ensino de Estatística.

Nesta mesma direção, a Educação a Distância aparece como alternativa para ampliação dos alcances das aulas de Estatística. Embora as pesquisas analisadas nessa temática (D01, D02 e D11) apontem alguns avanços, há muito a ser feito para que as características acima citadas e desejáveis para os processos de ensino e aprendizagem de Estatística estejam presentes também nessa modalidade de ensino, respeitando suas particularidades e os diversos níveis escolares em que a Estatística se faz presente.

Por fim, cabe destacar que parece consensual entre as pesquisas que o ensino de Estatística e Probabilidade deve estar sustentado num processo de investigação no qual o contexto atribua significado às análises realizadas 
e consistência aos conceitos e ideias explorados. Dessa forma, acreditamos que os recursos tecnológicos concorrem com esta finalidade, à medida que possibilitam simulações de experimentos complexos e/ou inviáveis em outros contextos, assim como a explicitação de estruturas, ideias e conceitos subjacentes às análises estatísticas, mas fundamentais à sua compreensão e à mobilização do pensamento estatístico. Estes são os princípios que, acreditamos, devem orientar as pesquisas e ações na busca pelo aprimoramento da Estatística enquanto campo da educação formal com o auxílio de recursos tecnológicos, sempre que conveniente.

\section{Referências}

[1] D. P. Ausubel. A aprendizagem significativa: a teoria de David Ausubel. São Paulo: Moraes, 1982.

[2] C. Batanero. Didáctica de la Estadística. Granada: Universidade de Granada, 2001.

[3] C. Batanero, J. D. Godino, M. J. Cañizares. Simulation as a tool to train pre-service school teachers. In Proceedings of First ICMI African Regional Conference. Johannesburg: ICMI. CDROM. 2005.

[4] M. Borovcnik, R. Kapadia. Research and Developments in Probability Education. International Electronic Journal of Mathematics. 4(3): 111130, 2009.

[5] G. W. Bright, D. Frierson, J. E. Tarr, C. Thomas. Navigating Through Probability in Grades 6-8. Reston, VA: NCTM, 2003.

[6] G. Burril. Statistics Education and the Role of Technology. Disponível em: $<$ http://math.unipa.it/ grim/EBurrill95104.PDF>. Acesso em 23 nov 2012.

[7] A. P. Canavarro. Estatística e Calculadoras Gráficas. In: C. Loureiro, F. Oliveira, L. Brunheira. (Orgs.) Ensino e Aprendizagem da Estatística. Lisboa: GRAFIS, páginas 159-167, 2000.

[8] T. M. M. Campos, I. M. Cazorla, V. Y. Kataoka. Statistics School Curricula in Brazil. In: C. Batanero, G. Burril, C. Reading. (Eds.). Teaching Statistics in School Mathematics-Challenges for Teaching and Teacher Education. London: Springer, páginas 5-8, 2011.

[9] B. Chance, D. Ben-Zvi, J. Garfield, E. Medina. The role of technology in improving student learning of statistics. Technology Innovations in Statistics Education Journal, 1(1):1-26, 2007.
[10] G. W. Cobb. The introductory statistics course: a ptolemaic curriculum? Technology Innovations in Statistics Education, 1(1): 3-17, 2007.

[11] F. R. Curcio. Developing graph comprehension: elementary and middle school activities. Reston: NCTM, 1989.

[12] R. Duval. Les différents fonctionnements possibles d'une figure dans une demarche géométrique. Repères, 17(1): 121-138, 1994.

[13] R. Duval. Registros de Representação Semiótica e funcionamento cognitivo da compreensão em Matemática. In: S. D. A. Machado, (Org.). Aprendizagem em Matemática: registros de representação semiótica. Campinas: Papirus, páginas 11-33, 2003.

[14] J. O. Freitas. A Estatística e as Novas Tecnologias. In: C. Loureiro, F. Oliveira, L. Brunheira. (Orgs.) Ensino e Aprendizagem da Estatística. Lisboa: GRAFIS, páginas 147-158, 2000.

[15] Gal, I., 2005. Towards "probability literacy" for all citizens: building blocks and instructional dilemas. In G. Jones (Ed.), Exploring Probability in Schools: Challenges for Teaching and Learning, pp- 39-63. Springer, New York.

[16] J. D. Godino, F. Ruiz, R. Roa, J. L. Pareja. A. M. Recio. Analysis of two internet interactive applets for teaching statistics in schools. In Proceedings of the Satellite Conference Statistics Education and the Internet. Granada, páginas 110, 2003.

[17] V. M. Kenski. Aprendizagem Mediada pela Tecnologia. Revista Diálogo Educacional, Curitiba, 4(10): 47-56, 2003.

[18] P. Lévy. As tecnologias da inteligência: o futuro do pensamento na era da Informática. Rio de Janeiro: Editora 34, 1993.

[19] C. C. Oliveira, J. W. Costa, M. Moreira. Ambientes informatizados de Aprendizagem: produção e avaliação de software educativo. São Paulo: Papirus, 2001.

[20] S. Papert. A máquina das crianças: repensando a escola na era da informática. Porto Alegre: Artes Médicas, 1994.

[21] J. P. Ponte, J. Brocardo, H. Oliveira. Investigações Matemáticas na sala de aula. Belo Horizonte: Autêntica, 2006.

[22] O. K. Tikhomirov, (Ed.) Man and computer. Moscow: Moscow University Press, 1972.

[23] J. A. Valente. A espiral da aprendizagem e as 
Tecnologias de Informação e Comunicação: repensando conceitos. In: M. C. R. A. Joly. A tecnologia no ensino: implicações para aprendizagem. São Paulo: Casa do Psicólogo, páginas 15-37, 2002.

[24] J. A. Valente. Computadores e conhecimento: repensando a educação. Campinas: Gráfica Central da UNICAMP, 1993.

[25] G. Vergnaud. La theorie des champs conceptuels. In: BRUN, J. (Org.). Didactique des Mathématiques. Lausanne: Delachaus; Paris: Nietlé, 1996. 